\title{
INCIDENTAL RADIOGRAPHIC FINDINGS AND THEIR RESTORATIVE IMPLICATIONS
}

\author{
R. Sridevi, Sai Laxman Bharadwaj B, R. Thirumalai Prabhu
}

1. Senior lecturer, Department of Prosthodontics, Adhiparasakthi Dental College and Hospital. Melmaruvathur, Tamil Nadu.

2. Intern, Department of Orthodontics, Adhiparasakthi Dental College and Hospital. Melmaruvathur, Tamil Nadu.

3. Intern, Department of Orthodontics, Adhiparasakthi Dental College and Hospital. Melmaruvathur, Tamil Nadu.

\section{CORRESPONDING AUTHOR}

Dr. R. Sridevi,

A-11, Laxmi Apartments,

Meenambal ST, Melmaruvathur, ,

Tamilnadu, India,

E-mail: itsalwayssreedevi@gmail.com,

Ph: 9109952363999.

ABSTRACT: Radiographs are an irreplaceable diagnostic tool, especially in dealing with dentofacial hard tissues. Guidelines like the ALARA require professionals to limit the number of radiographs prescribed to patient, thereby minimizing radiation dose. On the other hand, for prosthetic evaluation, a latest radiograph has the potential to uncover new findings that can alter the treatment planning sequence or affect the outcome of the planned treatment. This review article discusses ten such radiographs. The significant findings in each radiograph have been highlighted and treatment protocols tailored to the same. The aim of this article is to help the reader adopt a meticulous approach and a keen eye for detecting problems, and emphasizes the efficacy of radiographs in patient evaluation for restorative care.

KEYWORDS: Incidental, Findings, Treatment, Interpretation

INTRODUCTION: Dental clinics here in our country are teeming with patients keeping dental personnel on a tight schedule. Efficient practice in this scenario demands a good balance between speed, time management and prescribing what is needed and best for the patient. Frequently patients present for prosthetic rehabilitation with radiographs taken during previous treatment sittings and we often avoid prescribing a new one. Considering the possibility of incidental findings, it is wise practice to prescribe a new radiograph during abutment evaluation. This additional measure avoids unnecessary failures and the need for prosthetic retreatment like the removal and re-fabrication of fixed bridges which can cause considerable damage to both tooth structure and the patients' wallet.

All the radiographs reviewed here, presented to the Prosthodontic clinic in our institution for rehabilitation. A thorough perusal of this article will tell us how, and bring to light a multitude of possibilities and occurrences during diagnostic radiography; for what the mind does not know, the eyes do not see! 


\section{Radiograph 1 - RESIDUAL ROOT FRAGMENT}

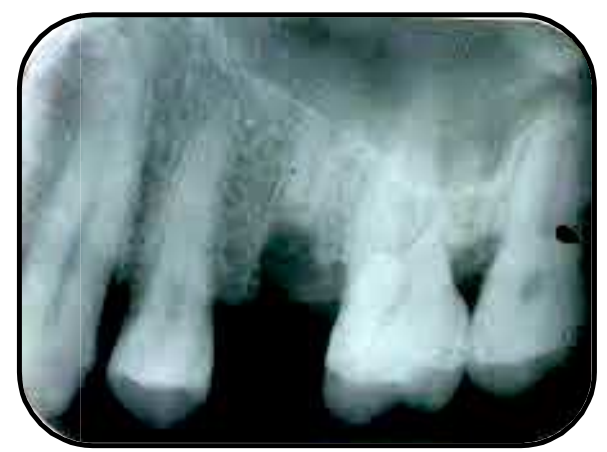

Fig 1

Fracture of a root during exodontia, particularly long,divergent ones is a common complication ${ }^{1}$. The edentulous area in this radiograph reveals the presence of a root fragment following extraction of the maxillary left second premolar. This incidental finding is familiar even with novice dental practitioners and cannot be missed. The fragment in this radiograph is more than $5 \mathrm{~mm}$ in length.

Current guidelines suggest that a root fragment of up to $5 \mathrm{~mm}$ can be left in situ in a healthy patient provided there is no evidence of apical pathology².

\section{Radiograph 2 - ADJACENT TOOTH PATHOLOGY}

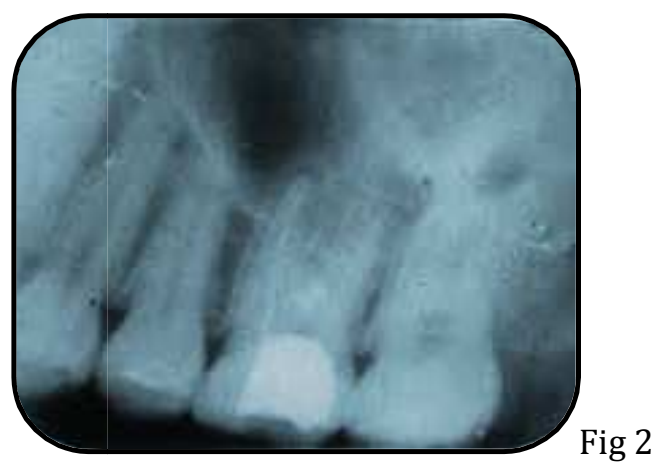

In endodontic practice, pulp sensibility testing of the tooth in question is corroborated with the response of control teeth which are usually adjacent teeth. These tests are indirect and subject to false positive and false negative readings ${ }^{3}$. In such cases, careful evaluation and interpretation of radiographic findings are an invaluable adjunct to pulp sensibility testing. In the above radiograph, exposed to evaluate 26 for crown placement, a periapical lesion was identified in relation to 25 . Clinical examination was positive for the presence of an occlusal Class I composite filling, tenderness to percussion and poor response to electric pulp testing when compared to the contra lateral 15. Endodontic treatment of 25 prior to restoration of 26 was considered prudent to avoid patient discomfort and minimize the number of prosthetic visits. 


\section{Radiograph 3 - MISSED ROOTS}

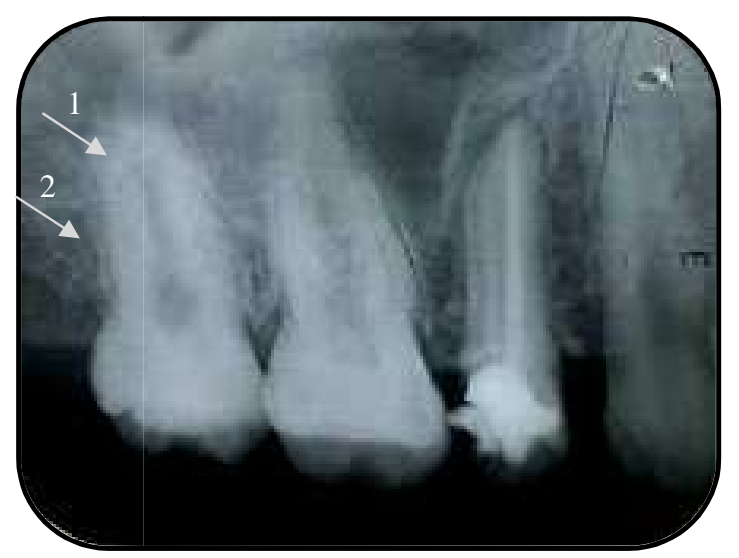

Fig 3

The root canal treated tooth approximating the maxillary right first molar in this radiograph shows clear evidence of two root outlines (white arrows)

The case notes for the patient under reference reveal that this tooth was designated as 15. However, obtaining the past dental history elicits extraction of the upper second premolars for orthodontic purposes. A maxillary first premolar has two canals one each in buccal and palatal roots, whereas a maxillary second premolar has a single root with a single canal ${ }^{4}$. In effect the tooth in question was a first premolar and was scheduled for orthograde retreatment.

This radiograph is significant for two other findings

1. The orientation dot has been positioned mesio-apical instead of mesio-occlusal

2. The restorative margin in the first premolar is at the level of the interdental bone distally due to tipping. Adjunctive periodontal therapy is imminent to ensure healthy placement of crown margins for long-term success.

\section{Radiograph 4 - CORONAL SEAL}

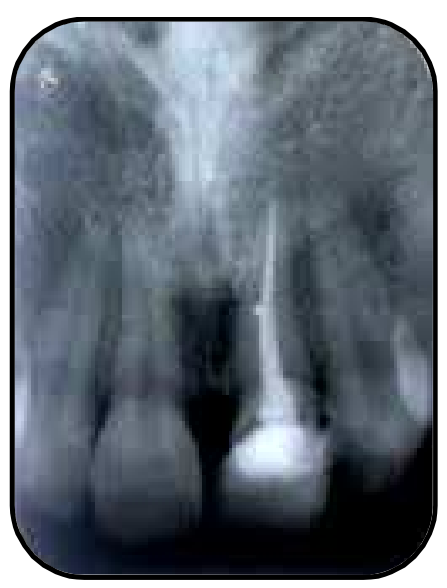

Fig 4 
Hermetic apical seal for eras has been considered the gold standard for endodontic success. Contemporary concepts stress that both the coronal and apical seal play an equal role in determining the same. Coronal seal refers to the protection rendered by a post endodontic restoration (direct or indirect). If the coronal seal is not intact, leakage through the dentinal tubules can establish reinfection within three months in the canal system leading to failure 5 .

The temporary restoration shown in the radiograph does not seal and cover the coronal portion adequately. Though marginal discrepancies can be detected clinically on facial and lingual aspects, the presence and extent of such defects in the approximal regions are detected with relative ease and precision on radiographs during follow-up visits. Also, the restored tooth in this radiograph is a victim of proximal (distal) under preparation and an over contoured crown (arrow).The necessary adjustments have to done and the crown has to be replaced at the earliest.

\section{Radiograph 5 - ROOT FRACTURE}

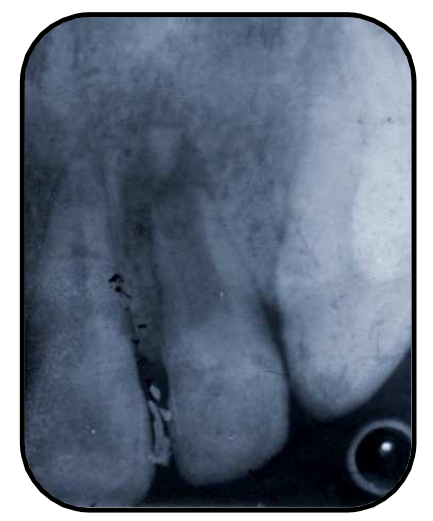

Fig 5

This is a pretreatment radiograph for a maxillary anterior diastema closure.

Transverse root fractures can occur in the coronal, middle and apical third. Apical third fractures are usually asymptomatic, exhibit no mobility and advocate a wait and watch approach for treatment ${ }^{6}$.

Apical root fractures are by and large detected on radiographs as depicted here in tooth 22 . Prosthetic treatment planning for such teeth involves

1. Eliciting the history of trauma

2. Thorough sensibility testing for all the teeth in the segment

3. Adjuvant endodontic therapy where mandatory

4. In case these teeth are to be used as fixed partial denture abutments, it should be borne in mind that there is an effective reduction in pericemental area available for support, especially in a weak abutment like the lateral incisor. 


\section{Radiograph 6 - CYSTIC LESIONS}

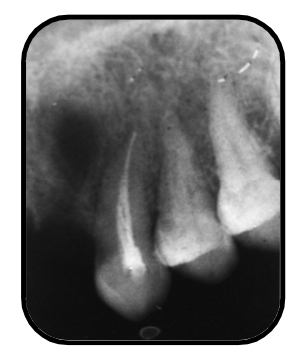

Fig 6

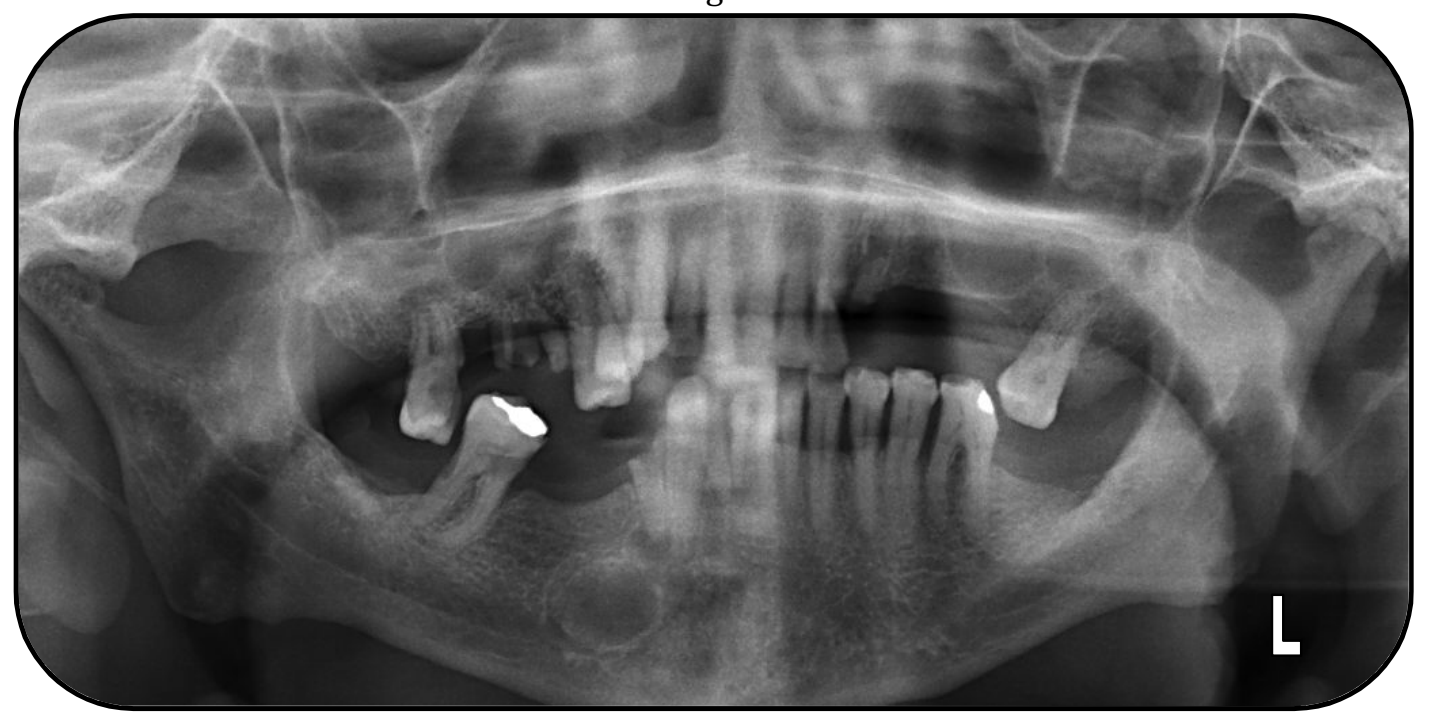

Fig 7

Cystic lesions in the jaws are generally incidental findings on radiographs. The extent and the nature of the lesion determine the prognosis. Henceforth, it is wise to subject these lesions to histopathological examination before harping on a treatment plan for the patient.

The periapical radiograph and the ortho-pantomograph are those of two different patients. The anterior maxillary lesion in the periapical (Fig 6) revealed a residual cyst. The right mandibular posterior lesion in the panoramic view (Fig 7) turned out to be an odontogenic keratocyst.

Significance - Residual cyst can expand and interfere with the fit of a prosthesis ${ }^{7}$, and the presence of this multilocular odontogenic keratocyst presents an absolute contraindication to implant placement or immediate replacement with fixed bridges owing to their aggressive behavior and high recurrence rate ${ }^{8}$. The residual cyst has to be thoroughly enucleated and following the period of healing definitive prosthesis can be fabricated. As far as the Gorlins cyst goes, it demands radical treatment and an extended follow up period to ensure complete regression of the lesion before any form of definitive rehabilitation is planned. 


\section{Radiograph 7 - FURCATION INVOLVEMENT}

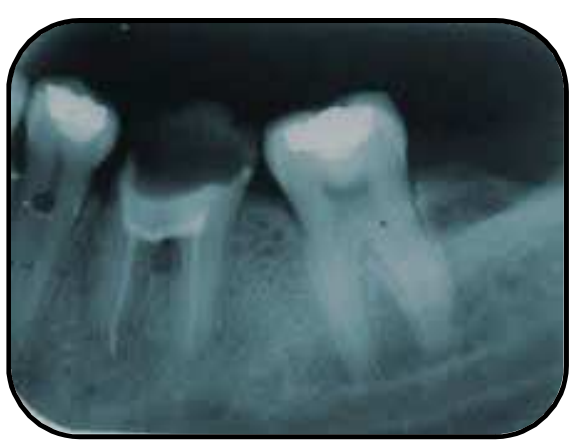

Fig 8

The pulp and the periodontium have an embryologic, anatomic and functional interrelationship. When the two are involved in disease simultaneously it is known as an endoperio lesion which classically can be demonstrated by furcation involvement of an endodontically compromised or previously root treated tooth.

The radiograph depicts a Class III furcation involvement in 36 and has the potential to be treated by surgical periodontal therapy ${ }^{9}$, but a close examination of the radiograph reveals

1) Poor quality endodontic therapy

2) Apical overextension of gutta percha in mesial root

3) Grossly destroyed coronal tooth structure

4) Class I restorations in the adjacent teeth

Taking into account all the above factors, the consensus was to extract the tooth and replace with a fixed partial denture with support from 35 and 37.

\section{Radiograph 8 - POST LENGTH}

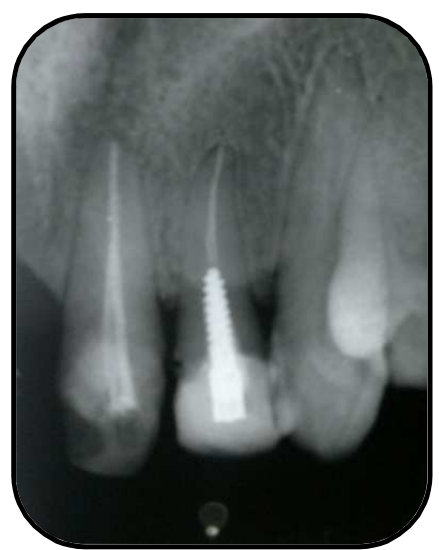

Fig 9

Anterior teeth are strategically located and designed to withstand the lateral forces. Post endodontic restoration of extensively damaged crowns warrants the use of a post for retention of the core. 
In selecting posts for anterior teeth, it is vital that the post should be long enough to satisfy clinical requirements without jeopardizing the root integrity ${ }^{10}$. The standard parameters are ${ }^{11}$,

1) The post should be $2 / 3^{\text {rd }}$ the length of the root

2) The length of the post can also equal the coronal length of the tooth

3) The post should extend into one half the length of the root embedded in its supporting bone.

In this patient the core in 22 is supported by a screwed post that does not extend to the adequate depth. The bone levels are well below normal and the post stops at the level of alveolar support. Short, stiff posts transfer forces to the unsupported root extending above the alveolus and can cause root fracture ${ }^{11}$. It was decided to use only the central incisor 21 as a terminal abutment to restore the edentulous area and follow up on the prognosis of the lateral incisor.

\section{Radiograph 9 - ANKYLOSED TOOTH}

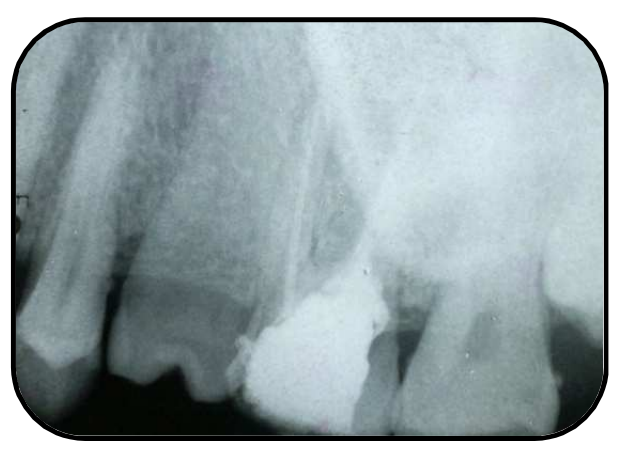

Fig 10

The patient presented for single unit restoration of root canal treated 26.

Accidental finding includes ankylosed maxillary left second premolar as evident in the absence of a distinct periodontal ligament space, definitive pulp canals and chamber and infraoccluded position.

Ankylosis is a pathologic fusion of the cementum or dentin of a tooth root to the alveolar bone ${ }^{12}$. Adults, with their slower rate of replacement resorption may retain an ankylosed tooth for many years with minimal treatment or minor cosmetic modifications ${ }^{13}$. At present, there are no guidelines in the dental literature for the treatment of ankylosis ${ }^{14}$. The parameters that influence treatment in this case include

1) Adult presentation and diagnosis of ankylosis

2) Mild infraocclusion

3) Considerable rotation and misplaced proximal contacts which necessitates pulp therapy before preparation for full coverage restorations.

4) An adjacent root treated molar

Therefore, it was decided to restore the molar with a full metal coverage crown combined with a minimal preparation onlay restoration on 25 to restore function.

CONCLUSION: A patient's radiograph is an indicator of both past and current disease experience. Dental professionals should aim at absorbing as much information as possible from 
a given radiograph, for, this information may possess the power to reroute our original treatment plan in lieu of the patient's best interests.

An incidental finding is one that accompanies and does not constitute the major reason for exposing the radiograph. Majority of these findings can be attributed to iatrogenic causes except for pathologies like cysts and fractures. Timely identification of these errors can best avoid irreversible damage to the dentition.

\begin{tabular}{|l|}
\hline What this article tells us? \\
\hline $\begin{array}{l}\text { To always consider the possibility of spotting pathologies on } \\
\text { routine radiography }\end{array}$ \\
\hline Why is it important? \\
\hline $\begin{array}{l}\text { The preservation of that which remains is of utmost importance } \\
\text { than the meticulous replacement of that which has been lost. }\end{array}$ \\
\hline How can we identify them? \\
\hline A thorough knowledge combined with adequate experience \\
\hline Where to learn these skills from? \\
\hline $\begin{array}{l}\text { Practice is the key. } \\
\text { It is the onus of every practitioner to keep himself up-to-date on } \\
\text { changing trends in treatment planning and diagnosis and apply } \\
\text { them on a daily basis. }\end{array}$ \\
\hline
\end{tabular}

\section{REFERENCES :}

1. Peterson. Ellis. Hupp. Tucker: Ch 11 Prevention and management of surgical complication in Contemporary oral and maxillofacial surgery, 4 ed, Pg 221-237

2. Abhay N Dhatarkar: Ch 13 in Exodontia Practice 1ed, Pg 129-134

3. V Gopikrishna et al; Assessment of pulp vitality - A review. International Journal of Pediatric Dentistry 2009, Vol 19: 3-15.

4. T.R Pitt Ford, Ch 3 in Endodontics- Problem Solving In Clinical Practice, Pg 33-35

5. B A Begotka et al, The importance of coronal seal following root canal treatment. Virginia Association Dental Journal, 1996, Oct-Dec, 73(4); 8-10

6. J.O Andreasen and F.M Andresen: Ch 12 Root fractures in Text Book and Color Atlas of Traumatic Injuries to the teeth 4ed, pg 337-371

7. Cawson and Odell: Cysts of the jaws in Essentials of Oral Pathology and Medicine 7ed, Pg 102-128

8. Nakamura et al: Marsupilization of Odontogenic Keratocysts - Long term follow up analysis of the effect and changes in growth characteristics, Oral Surgery Oral Medicine Oral Pathology Radiology Endodontology, 2002 Vol 94: 543-553.

9. Murray Arlin, Oral Health 1987 Vol 77(5): 29-34 
10. Goodacre CJ et al: The Prosthodontic Management of Endodontically treated teeth - A literature review II - Maintaining the apical seal, Journal of Prosthetic Dentistry 4:51, 1995

11. Stephen Cohen, Ch 21 Restoration of Endodontically treated teeth in Pathways of the Pulp, Pg $786-821$

12. William Biederman et al, Etiology and treatment of tooth ankylosis, American Journal of Orthodontics, Vol 48(9): 670-684

13. Ebeleseder KA, Friehs S, et al, A study of replanted permanent teeth in different age groups. Endod Dent Traumatol 14(6):274-8, 1998

14. Moffat MA et al , Intentional surgical repositioning of ankylosed permanent maxillary incisor Dental Traumatology 2002; 18: 222-226

Journal of Evolution of Medical and Dental Sciences/Volume1/ Issue3/July-Sep $2012 \quad$ Page 280 